\title{
Deformable Porous Media with Double Porosity. Quasi-Statics. II: Memory Effects
}

\author{
J. L. AURIAULT \\ Institut de Mécanique de Grenoble, UMR 101, Université Joseph Fourier, \\ Institut National Polytechnique de Grenoble, CNRS, BP 53 X, 38041 Grenoble Cedex, France \\ and \\ C. BOUTIN \\ Ecole Nationale des Travaux Publics de lEtat, Laboratoire Géomatériaux, rue M. Audin, \\ 69518 Vaulx en Velin Cedex, France
}

\begin{abstract}
As in our previous paper, we investigate the macroscopic quasi-static description of a porous medium with a double porosity constituted by pores and fractures. For this purpose, we use a homogenization technique. As expected, the macroscopic description is sensitive to the ratios between the different scales, $l / l^{\prime}$ and $l^{\prime} / l^{\prime \prime}$ where $l, l^{\prime}, l^{\prime \prime}$ are characteristic lengths of the pores, the fractures, and the macroscopic medium, respectively. In the first paper, we investigated the case $l^{\prime} / l^{\prime \prime}=O\left(l^{2} / l^{2}\right) \ll 1$ (case 1) which exhibits a coupling between the flows through the pores and the fractures. In the present paper, we deal with the other homogenizable cases. The case 3 where $l / l^{\prime}=O\left(l^{\prime 2} / l^{\circ 2}\right) \ll 1$ gives a macroscopic description similar to that of a single porosity medium. The main result, however, is the case 2, where $l / l^{\prime}=O\left(l^{\prime} / l^{\prime \prime}\right) \ll 1$, which exhibits memory effects. These are due to the seepage through the micropores.
\end{abstract}

Key words. Deformable porous media, double porosity, consolidation, memory effect.

\section{Nomenclature}

\section{Roman Letters}

a

$\mathbf{c}^{\prime}, \mathbf{c}^{\prime \prime}$

D

e

$f$

$\mathbf{k}_{p}, \mathbf{k}_{f}$

$\mathbf{K}_{p}, \mathbf{K}_{f}$

I

$l, l^{\prime}, l^{\prime \prime}$

$n, n^{\prime}$

elastic tensor of the skeleton material

effective elastic tensors of the skeleton

rate of deformation tensor

deformation tensor

subscript for the fractures

particular solutions for the velocity field $\mathbf{v}$ in the pores and the fractures, respectively

filtration tensors of the pores and the fractures, respectively

unit tensor

characteristic lengths of the pore scale, the fracture scale and the macroscopic medium, respectively

pore porosity and fracture porosity, respectively

normal unit vector 
$p_{p}, p_{f} \quad$ pressures in the pores and the fractures, respectively subscript for the solid characteristic times for the pore and fracture flows, respectively solid, pore and fracture fluid displacements, respectively solid, pore and fracture fluid velocities, respectively space variables for the pore, fracture and macroscopic scales, respectively

Greek Letters $\alpha^{\prime}, \alpha^{\prime \prime}, \gamma^{\prime}, \gamma^{\prime \prime}$ $\beta^{\prime}, \beta^{\prime \prime}$

coupling effective tensors entering the behavior relations of the porous medium

\section{$\sigma_{s}, \sigma_{p}, \sigma_{f}$}

$\tau, \tau^{\prime}$

$\Omega, \Omega^{\prime}$ $\Omega_{s}, \Omega_{p}, \Omega_{s p}^{\prime}, \Omega_{f}^{\prime}$ coupling effective scalars entering the behavior relations of the porous medium particular solution for the pressure field boundaries of the pores and the fractures, respectively Laplace operator gradient operator small parameter of the homogenization process particular solutions for the displacement field $\mathbf{u}$ particular solutions for the displacement field $\mathbf{u}$ pulsation density of the fluid viscosity particular solutions for the displacement field $\mathbf{u}$ solid, pore and fracture fluid stress tensors, respectively particular solutions for the pressure periods at the pore and fracture scales, respectively parts of the periods occupied by the solid, the pores, the solid plus the pores and the fractures, respectively

Particular Symbols

$\langle\Phi\rangle_{\Omega},\langle\Phi\rangle_{\Omega^{\prime}},\langle\Phi\rangle_{\Omega \Omega^{\prime}}$ volume averages of the quantity $\Phi$ on $\Omega, \Omega^{\prime}$, and $\Omega$ plus $\Omega^{\prime}$, respectively

\section{Introduction}

As in our previous paper (Auriault and Boutin, 1991 - paper I), we considered a deformable porous medium made of two porosity systems undergoing a quasi-static excitation. $l, l^{\prime}$ and $l^{\prime \prime}$ are characteristic lengths of the pores, the fractures, and the macroscopic medium, respectively. In paper $\mathrm{I}$, the case where $l^{\prime} / l^{\prime \prime}=O\left(l^{2} / l^{2}\right) \ll 1$ was investigated and the macroscopic description was obtained from the descriptions at the pore and fracture levels by using an homogenization technique. For 
such a situation, referred to as case 1 , a coupling exists between the flows through the porous and fractured media. The macroscopic description was shown to be similar to the one given by M. A. Biot:

$$
\begin{aligned}
& \nabla^{\prime \prime}\left(\mathbf{c}^{\prime \prime} \mathbf{e}^{\prime \prime}\left(\mathbf{u}_{s}^{0}\right)-\alpha^{\prime \prime} p^{0}\right)=0, \\
& \nabla^{\prime \prime}\left(\left\langle\mathbf{v}_{f}^{0}\right\rangle_{\Omega^{\prime}}-n^{\prime} \mathbf{v}_{s}^{0}\right)=-\gamma^{\prime \prime} \frac{\partial \mathbf{e}^{\prime \prime}\left(\mathbf{u}_{s}^{0}\right)}{\partial t}-\beta^{\prime \prime} \frac{\partial p^{0}}{\partial t}, \\
& \left\langle\mathbf{v}_{f}^{0}\right\rangle_{\Omega^{\prime}}-n^{\prime} \mathbf{v}_{s}^{0}=-\mathbf{K}_{f} \nabla^{\prime \prime} p^{0},
\end{aligned}
$$

but with a broken symmetry

$$
\gamma^{\prime \prime} \neq \alpha^{\prime \prime}
$$

Here $\mathbf{c}^{\prime \prime}, \alpha^{\prime \prime}, \gamma^{\prime \prime}$ and $\beta^{\prime \prime}$ are here elastic coefficients. As expected, the macroscopic permeability is that of only the fractures.

This paper is devoted to the investigation of other situations of interest, cases 2 and 3. For case $2, l / l^{\prime}=O\left(l^{\prime} / l^{\prime \prime}\right) \ll 1$, and the macroscopic equivalent description will again be shown to be similar to Biot's description but, for harmonic quasi-static excitations, with complex and frequency dependent effective coefficients. The porous medium therefore displays memory effects. The symmetry is broken as in case 1.

Case 3 is related to $l / l^{\prime}=O\left(l^{\prime 2} / l^{\prime \prime 2}\right) \ll 1$. The macroscopic description will appear as the classical Biot one where the matrix plus the pores behave like a monophasic elastic medium.

The three cases are shown in the following logarithmic diagram where the relative position of the three scales is shown in each case:

case 1: $\log l \quad . \quad \log l^{\prime} \quad . \quad . \quad . \log l^{\prime \prime}$

case 2: $\log l$. . $\quad \log l^{\prime} \quad . \quad . \log l^{\prime \prime}$

case 3: $\log l$. . . $\quad \log l^{\prime} . \log l^{\prime \prime}$

It should be remarked that the permeabilities $\mathbf{K}_{p}$ and $\mathbf{K}_{f}$ of the pores and the fractures, respectively, make it possible to estimate the ratio $l / l^{\prime}$ which is a measure of the separation between the pore and fracture scales. We have the following rough approximations

$$
\mathbf{K}_{p}=O\left(\frac{l^{2}}{\mu}\right), \quad \mathbf{K}_{f}=O\left(\frac{l^{\prime 2}}{\mu}\right) .
$$

Therefore, the separation of the two scales is given by

$$
\frac{1}{l^{\prime}}=O\left(\sqrt{\frac{\mathbf{K}_{p}}{\mathbf{K}_{f}}}\right) .
$$


Contrary to this, the ratio $l^{\prime} / l^{\prime \prime}$ cannot be obtained from the properties of the medium, since $l^{\prime \prime}$ characterizes either the excitation or the size of an appropriate macroscopic sample.

As recalled above, the excitations are assumed to be quasistatic: the inertial effects are neglected. This corresponds to small velocities, so that the inertial convective terms are small. For the transient inertial effects, this implies that the transient Reynolds number is small at the lower scale $\rho \omega l^{2} / \mu \ll 1$.

The notations are those of paper I and the reader will refer to this paper and to Auriault, (1991) for the methodology to be used in what follows. The geometry of the medium is recalled in Figures 1 and 2.

Sections 2 and 3 are devoted to cases 2 and 3, respectively. After this, a recapitulation is presented in Section 4.

In order to avoid cumbersome developments, as in paper I we simplify the medium to an elastic porous matrix filled by an incompressible and viscous Newtonian fluid. All demonstrations of the existence and uniqueness for the boundary-value problems are also disregarded.

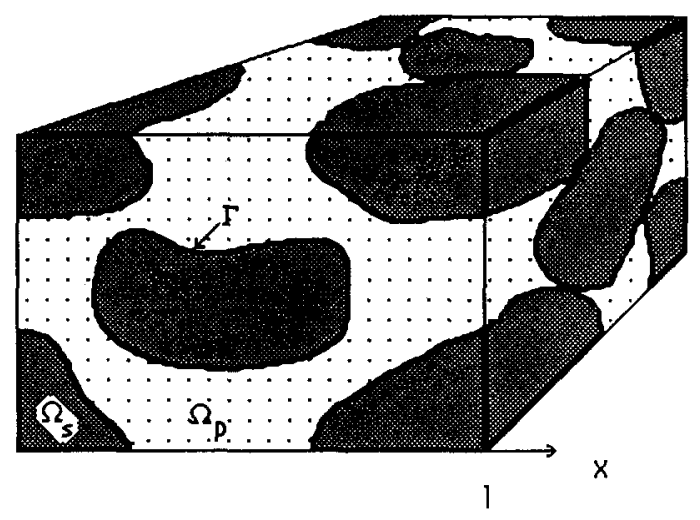

Fig. 1. Periodic cell of the microporous medium.
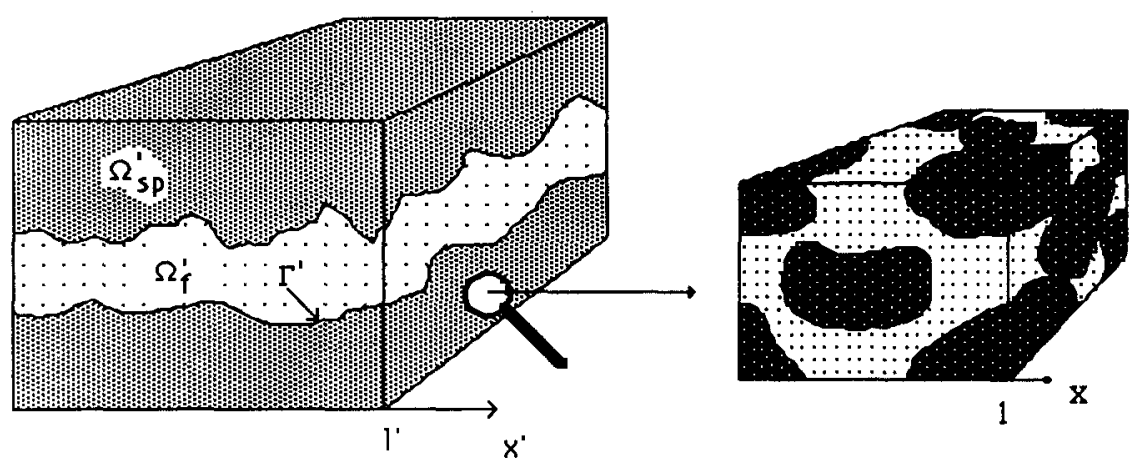

Fig. 2. Periodic cell of the fractured medium. 


\section{Case 2}

We consider a double porosity medium where the scales with characteristic lengths $l, l^{\prime}$, and $l^{\prime \prime}$ are identically separated:

$$
\frac{l}{l^{\prime}}=O\left(\frac{l^{\prime}}{l^{\prime \prime}}\right)=\varepsilon \ll 1
$$

The three characteristic lengths introduce three space variables $\mathbf{x}, \mathbf{x}^{\prime}$ and $\mathbf{x}^{\prime \prime}$ related between them by powers of $\varepsilon$ :

$$
\mathbf{x}=\varepsilon^{-2} \mathbf{x}^{\prime \prime}, \quad \mathbf{x}^{\prime}=\varepsilon^{-1} \mathbf{x}^{\prime \prime} .
$$

As in paper I, it is also possible to introduce two characteristic times $T_{p}$ and $T_{f}$ for the flows through the pores and the fractures, respectively. Here again, they are related by

$$
\frac{T_{f}}{T_{p}}=\mathbf{O}\left(\frac{l^{2}}{l^{\prime 2}}\right)=\mathbf{O}\left(\varepsilon^{2}\right)
$$

As a consequence, by following the procedure defined in paper I, each unknown $\Phi$ will be looked for in the form

$$
\begin{aligned}
& \Phi=\Phi^{0}\left(\mathbf{x}, \mathbf{x}^{\prime}, \mathbf{x}^{\prime \prime}, t, T\right)+\varepsilon \Phi^{1}\left(\mathbf{x}, \mathbf{x}^{\prime}, \mathbf{x}^{\prime \prime}, t, T\right)+\cdots, \\
& \mathbf{x}=\varepsilon^{-2} \mathbf{x}^{\prime \prime}, \quad \mathbf{x}^{\prime}=\varepsilon^{-1} \mathbf{x}^{\prime \prime}, \quad T=\varepsilon^{2} t,
\end{aligned}
$$

$\Phi^{i} \Omega$ and $\Omega^{\prime}$ periodic. Equations (1) induce the choice of $\mathbf{x}^{\prime \prime}$ and $t$ as the driving space and time variable, respectively. Therefore, equations are to be normalized by using $l^{\prime \prime}$ and $T_{f}$ as the characteristic length and time, respectively.

\subsection{NORMALIZATION}

We recall the descriptions at the microscales, as given by Equations (3)-(8) of paper I:

In the solid matrix, the equations are the quasi-static momentum balance:

$$
\nabla \sigma_{s}=0
$$

and the constitutive law (infinitesimal elasticity)

$$
\sigma_{s}=\mathbf{a e}\left(\mathbf{u}_{s}\right),
$$

where the stress $\sigma_{s}$ is related to the small deformation

$$
\mathbf{e}\left(\mathbf{u}_{S}\right)=\frac{1}{2}\left(\nabla+{ }^{t} \nabla\right) \mathbf{u}_{S}
$$

by the elastic tensor $\mathbf{a}$.

In the fluid, we have the quasi-static momentum balance

$$
\mu \Delta \mathbf{v}_{\alpha}-\nabla p_{\alpha}=0, \quad \sigma_{\alpha}=-p_{\alpha} \mathbf{I}+2 \mu \mathbf{D},
$$


where $\mu$ is the viscosity, $\mathbf{v}$ the velocity, $p$ the pressure, and $\mathbf{D}$ the rate of deformation: $\mathbf{D}=\mathbf{e}(\mathbf{v})$. The subscript $\alpha$ takes the values $p$ and $f$ in the pores and the fractures, respectively.

The incompressibility is written

$$
\nabla \mathbf{v}_{\alpha}=0, \quad \alpha=p, f .
$$

The boundary conditions on $\Gamma$ are the continuity of the normal stresses and the velocities

$$
\sigma_{s} \mathbf{N}=\sigma_{p} \mathbf{N} \text { on } \Gamma,
$$

where $\mathbf{N}$ is a unit normal to $\Gamma$ and

$$
\mathbf{v}_{s}=\mathbf{v}_{p} \text { on } \Gamma \text {. }
$$

The dimensionless numbers $Q_{p}$ and $Q_{f}$ are defined as the ratios of the viscous term to the pressure term of the Stokes equations for the pores and the fractures, respectively. And we have now, when using $l^{\prime \prime}$ and $T_{f}$, to normalize

$$
Q_{p}=O\left(Q_{f}\right)=\frac{\mu \Delta \mathbf{v}_{\alpha}}{\nabla p_{\alpha}}=O\left(\frac{\mu \mathbf{u}_{p} l^{\prime \prime}}{l^{\prime \prime 2} T_{f} p}\right)=O\left(\frac{l^{2} T_{p}}{l^{\prime \prime 2} T_{f}}\right)=O\left(\varepsilon^{2}\right) .
$$

As a consequence, Equation (4) will be now normalized by

$$
\varepsilon^{2} \mu \Delta \mathbf{v}_{\alpha}-\nabla p_{\alpha}=0, \quad \alpha=p, f,
$$

and the corresponding constitutive laws are

$$
\sigma_{\alpha}=-p_{\alpha} \mathbf{I}+2 \varepsilon^{2} \mu \mathbf{D} .
$$

In conclusion, the normalized set is written in the form

$$
\begin{aligned}
& \nabla \sigma_{s}=0 . \\
& \sigma_{s}=\mathbf{a e}\left(\mathbf{u}_{s}\right) \text { in } \Omega_{s} . \\
& \varepsilon^{2} \mu \Delta \mathbf{v}_{\alpha}-\nabla p_{\alpha}=0, \\
& \sigma_{\alpha}=-p \mathbf{I}+2 \varepsilon^{2} \mu \mathbf{D}, \\
& \nabla \mathbf{v}_{\alpha}=0 \text { in } \Omega_{\alpha}, \alpha=p, f, \\
& \sigma_{s} \mathbf{N}=\sigma_{p} \mathbf{N}, \\
& \mathbf{u}_{s}=\mathbf{u}_{p} \text { on } \Gamma, \\
& p_{p}=p_{f}, \\
& \left(\left\langle\sigma_{s}\right\rangle_{\Omega}+\left\langle\sigma_{p}\right\rangle_{\Omega}\right) \mathbf{N}^{\prime}=\sigma_{f} \mathbf{N}^{\prime},
\end{aligned}
$$

and

$$
\mathbf{v}_{f}-\left\langle\mathbf{v}_{s}\right\rangle_{\Omega}=\left\langle\mathbf{v}_{p}\right\rangle_{\Omega} \text { on } \Gamma^{\prime}
$$


The reader should refer to paper I for the justification of the boundary conditions (16) and (17) on $\Gamma^{\prime}$.

The unknowns are looked for in the form of asymptotic developments like (1), taking into account the fact that in $\Omega_{f}^{\prime}$ the solution is $\mathbf{x}$ independent, except in possible boundary layers along $\Gamma^{\prime}$ where velocities and pressures in the pores and in the fractures are matched. For the case under consideration, the unknowns are in the form

$$
\begin{aligned}
& \mathbf{u}_{s}=\mathbf{u}_{s}^{0}\left(\mathbf{x}, \mathbf{x}^{\prime}, \mathbf{x}^{\prime \prime}, t, T\right)+\varepsilon \mathbf{u}_{s}^{1}\left(\mathbf{x}, \mathbf{x}^{\prime}, \mathbf{x}^{\prime \prime}, t, T\right)+\cdots, \\
& \mathbf{u}_{p}=\mathbf{u}_{p}^{0}\left(\mathbf{x}, \mathbf{x}^{\prime}, \mathbf{x}^{\prime \prime}, t, T\right)+\varepsilon \mathbf{u}_{p}^{1}\left(\mathbf{x}, \mathbf{x}^{\prime}, \mathbf{x}^{\prime \prime}, t, T\right)+\cdots, \\
& p_{p}=p_{p}^{0}\left(\mathbf{x}, \mathbf{x}^{\prime}, \mathbf{x}^{\prime \prime}, t, T\right)+\varepsilon p_{p}^{1}\left(\mathbf{x}, \mathbf{x}^{\prime}, \mathbf{x}^{\prime \prime}, t, T\right)+\cdots, \\
& \mathbf{u}_{f}=\mathbf{u}_{f}^{0}\left(\mathbf{x}^{\prime}, \mathbf{x}^{\prime \prime}, t, T\right)+\varepsilon \mathbf{u}_{f}^{1}\left(\mathbf{x}^{\prime}, \mathbf{x}^{\prime \prime}, t, T\right)+\cdots, \\
& p_{f}=p_{f}^{0}\left(\mathbf{x}^{\prime}, \mathbf{x}^{\prime \prime}, t, T\right)+\varepsilon p_{f}^{1}\left(\mathbf{x}^{\prime}, \mathbf{x}^{\prime \prime}, t, T\right)+\cdots, \\
& \mathbf{x}=\varepsilon^{-2} \mathbf{x}^{\prime \prime}, \quad \mathbf{x}^{\prime}=\varepsilon^{-1} \mathbf{x}^{\prime \prime}, \quad T=\varepsilon^{2} t, \\
& \mathbf{u}_{s}^{i}, \mathbf{u}_{p}^{i} \text { and } p_{p}^{i} \Omega \text { and } \Omega^{\prime} \text { periodic and } \mathbf{u}_{f}^{i}, p_{f}^{i} \Omega^{\prime} \text { periodic. }
\end{aligned}
$$

\subsection{MACROSCOPIC DESCRIPTION}

Introducing the above developments in the set (8)-(17), we obtain successive boundary-value cell problems for the different terms of the expansions. These problems are investigated in the Appendix and lead to the macroscopic equivalent description. This one is composed of two parts, i.e. a momentum balance and a mass balance.

The momentum balance concerns the bulk medium. When considering harmonic excitations of pulsation $\omega$, it is given by (A.26):

$$
\nabla^{\prime \prime}\left(\mathbf{c}^{\prime \prime} \mathbf{e}^{\prime \prime}\left(\mathbf{u}_{s}^{0}\right)-\alpha^{\prime \prime} p_{f}^{0}\right)=0
$$

where the tensors $\mathbf{c}^{\prime \prime}$ and $\alpha^{\prime \prime}$ are complex valued and $\omega$ dependent.

The mass balance is (A.27):

$$
\nabla^{\prime \prime}\left(\mathbf{K}_{f} \nabla^{\prime \prime} p_{f}^{0}\right)=-\gamma^{\prime \prime} i \omega \mathbf{e}^{\prime \prime}\left(\mathbf{u}_{s}^{0}\right)-\beta^{\prime \prime} i \omega p_{f}^{0}, \quad \mathbf{K}_{f}=\left\langle\mathbf{k}_{f}\right\rangle_{\Omega^{\prime}},
$$

where $\mathbf{K}_{f}$ is the permeability tensor of the fractures. $\gamma^{\prime \prime}$ and $\beta^{\prime \prime}$ are complex valued and $\omega$ dependent.

In conclusion, the macroscopic description of the double porosity medium of case 2, where

$$
\frac{l}{l^{\prime}}=O\left(\frac{l^{\prime}}{l^{\prime \prime}}\right)=\varepsilon \ll 1
$$

is given by (18) and (19). It exhibits a similar structure to Biot's. But, as in the case 1 , the symmetry is broken, $\gamma^{\prime \prime} \neq \alpha^{\prime \prime}$. This can be shown by using the same route as 
in case 1 (paper I). Moreover, the effective coefficients $\mathbf{c}^{\prime \prime}, \alpha^{\prime \prime}$, and $\beta^{\prime \prime}$ are here complex valued and $\omega$ dependent. Under transient excitations, the medium will show memory effects. The macroscopic description needs only the fracture pressure field. But in opposition to case 1, the pore pressure is here different from the pressure in the fractures.

\section{Case 3}

We now investigate case 3 where the scales are related by

$$
\frac{l}{l^{\prime}}=\varepsilon^{2}=O\left(\frac{l^{\prime 2}}{l^{\prime \prime 2}}\right) \ll 1
$$

The three space variables $\mathbf{x}, \mathbf{x}^{\prime}$ and $\mathbf{x}^{\prime \prime}$ to be considered are therefore linked by

$$
\mathbf{x}=\varepsilon^{-3} \mathbf{x}^{\prime \prime}, \quad \mathbf{x}^{\prime}=\varepsilon^{-1} \mathbf{x}^{\prime \prime} .
$$

The two characteristic times $T_{p}$ and $T_{f}$ for the flows through the pores and the fractures, respectively, are now such that

$$
\frac{T_{f}}{T_{p}}=O\left(\frac{l^{2}}{l^{\prime 2}}\right)=O\left(\varepsilon^{4}\right) .
$$

As a consequence, by following the procedure defined in paper I, each unknown $\Phi$ will be looked for in the form

$$
\begin{aligned}
& \Phi=\Phi^{0}\left(\mathbf{x}, \mathbf{x}^{\prime}, \mathbf{x}^{\prime \prime}, t, T\right)+\varepsilon \Phi^{1}\left(\mathbf{x}, \mathbf{x}^{\prime}, \mathbf{x}^{\prime \prime}, t, T\right)+\cdots, \\
& \mathbf{x}=\varepsilon^{-3} \mathbf{x}^{\prime \prime}, \quad \mathbf{x}^{\prime}=\varepsilon^{-1} \mathbf{x}^{\prime \prime}, \quad T=\varepsilon^{4} t,
\end{aligned}
$$

$\Phi^{i}, \Omega$ and $\Omega^{\prime}$ periodic. Again, the formulation (1) induces the choices of $\mathbf{x}^{\prime \prime}$ and $t$ as driving space and time variable, respectively. Therefore equations are to be normalized by using $l^{\prime \prime}$ and $T_{f}$ as the characteristic length and time, respectively.

\subsection{NORMALIZATION}

The descriptions at the microscales are given by Equations (2) - (7). The dimensionless numbers $Q_{p}$ and $Q_{f}$ are again defined by the following relations, when using $l^{\prime \prime}$ and $T_{f}$ to normalize

$$
Q_{p}=O\left(Q_{f}\right)=\frac{\mu \Delta \mathbf{v}_{\alpha}}{\nabla p_{\alpha}}=O\left(\frac{\mu \mathbf{u}_{p} l^{\prime \prime}}{l^{\prime \prime 2} T_{f} p}\right)=O\left(\frac{l^{2} T_{p}}{l^{\prime \prime 2} T_{f}}\right)=O\left(\varepsilon^{2}\right) .
$$

As a consequence, Equation (4) will be normalized, as in Section 2, by

$$
\varepsilon^{2} \mu \Delta \mathbf{v}_{\alpha}-\nabla p_{\alpha}=0, \quad \alpha=p, f
$$

and the corresponding constitutive laws are

$$
\sigma_{\alpha}=-p_{\alpha} \mathbf{I}+2 \varepsilon^{2} \mu \mathbf{D} \text {. }
$$


The normalized local description is therefore that of Section 2, i.e. Equations (8) $-(17)$. But the unknowns are now looked for in the form

$$
\begin{aligned}
& \mathbf{u}_{s}=\mathbf{u}_{s}^{0}\left(\mathbf{x}, \mathbf{x}^{\prime}, \mathbf{x}^{\prime \prime}, t, T\right)+\varepsilon \mathbf{u}_{s}^{1}\left(\mathbf{x}, \mathbf{x}^{\prime}, \mathbf{x}^{\prime \prime}, t, T\right)+\cdots, \\
& \mathbf{u}_{p}=\mathbf{u}_{p}^{0}\left(\mathbf{x}, \mathbf{x}^{\prime}, \mathbf{x}^{\prime \prime}, t, T\right)+\varepsilon \mathbf{u}_{p}^{1}\left(\mathbf{x}, \mathbf{x}^{\prime}, \mathbf{x}^{\prime \prime}, t, T\right)+\cdots, \\
& p_{p}=p_{p}^{0}\left(\mathbf{x}, \mathbf{x}^{\prime}, \mathbf{x}^{\prime \prime}, t, T\right)+\varepsilon p_{p}^{1}\left(\mathbf{x}, \mathbf{x}^{\prime}, \mathbf{x}^{\prime \prime}, t, T\right)+\cdots, \\
& \mathbf{u}_{f}=\mathbf{u}_{f}^{0}\left(\mathbf{x}^{\prime}, \mathbf{x}^{\prime \prime}, t, T\right)+\varepsilon \mathbf{u}_{f}^{1}\left(\mathbf{x}^{\prime}, \mathbf{x}^{\prime \prime}, t, T\right)+\cdots, \\
& p_{f}=p_{f}^{0}\left(\mathbf{x}^{\prime}, \mathbf{x}^{\prime \prime}, t, T\right)+\varepsilon p_{f}^{1}\left(\mathbf{x}^{\prime}, \mathbf{x}^{\prime \prime}, t, T\right)+\cdots, \\
& \mathbf{x}=\varepsilon^{-3} \mathbf{x}^{\prime \prime}, \quad \mathbf{x}^{\prime}=\varepsilon^{-1} \mathbf{x}^{\prime \prime}, \quad T=\varepsilon^{4} t, \\
& \mathbf{u}_{s}^{i}, \mathbf{u}_{p}^{i}, p_{p}^{i} \Omega \text { and } \Omega^{\prime} \text { periodic and } \mathbf{u}_{f}^{i}, p_{f}^{i} \Omega^{\prime} \text { periodic. }
\end{aligned}
$$

\subsection{MACROSCOPIC DESCRIPTION}

The way to be followed is similar to the one described in the Section 2.2. The macroscopic description is obtained by inserting the above expansions in the set (8) -(17) and by solving the successive boundary-value cell problems. The details of the calculus have not been provided in the paper because of the lack of space, but they can be obtained directly from the authors.

The macroscopic description is again composed of a momentum balance and a mass balance.

The momentum balance is

$$
\nabla^{\prime \prime}\left(\mathbf{c}^{\prime \prime} \mathbf{e}^{\prime \prime}\left(\mathbf{u}_{s}^{0}\right)-\alpha^{\prime \prime} p_{f}^{0}\right)=0,
$$

where the tensors $\mathbf{c}^{\prime \prime}$ and $\alpha^{\prime \prime}$ are now real valued.

The mass balance is

$$
\nabla^{\prime \prime}\left(\mathbf{K}_{f} \nabla^{\prime \prime} p_{f}^{0}\right)=-\gamma^{\prime \prime} \frac{\partial \mathbf{e}^{\prime \prime}\left(\mathbf{u}_{s}^{0}\right)}{\partial t}-\beta^{\prime \prime} \frac{\partial p_{f}^{0}}{\partial t}, \quad \mathbf{K}_{f}=\left\langle\mathbf{k}_{f}\right\rangle_{\Omega^{\prime}},
$$

where $\mathbf{K}_{f}$ is the permeability tensor of the fractures and $\gamma^{\prime \prime}$ and $\beta^{\prime \prime}$ are real tensors.

In conclusion, the macroscopic description of the double porosity medium of case 3, where

$$
\frac{l}{l^{\prime}}=\varepsilon^{2}=O\left(\frac{l^{\prime 2}}{l^{\prime \prime 2}}\right) \ll 1
$$

is given by (21) and (22). It exhibits a similar structure to Biot's. The macroscopic description needs only the fracture pressure field. But in opposition to case 1, and as in the case 2, the pore pressure is different from the pressure in the fractures. At the first order, there is no relative movement of the pore fluid to the matrix. The macroscopic behaviour of the matrix plus the fluid filled pores is that of a monophasic elastic medium. And the pore fluid does not participate in the seepage. 
Therefore, we recover, at the first order, a classical single porosity medium and the symmetry $\gamma^{\prime \prime}=\alpha^{\prime \prime}$ is verified. The fine pore porosity is ignored.

\section{Concluding Remarks}

The three cases investigated in papers I and II are the only situations where the double porosity plays an important part. It is easy to check that for $l^{\prime} / l^{\prime \prime}=O\left(l^{3}\right)$ $\left.l^{\prime 3}\right) \ll 1$, case $1^{*}$, we recover a classical, symmetrical Biot description, even if, due to the separation of scales $l / l^{\prime} \ll 1$, the macroscopic permeability is always the fracture permeability. Going further, the pore and fracture scales become nonseparated and the medium goes to a single porous medium.

In opposition to case $1^{*}$, case $3^{*}$ defined by $l^{\prime 3} / l^{\prime 3}=O\left(l / l^{\prime}\right) \ll 1$, is also modelled by the classical symmetrical Biot description where the pore plus matrix domain behaves like an elastic equivalent medium. All cases are summarized in the following logarithmic diagram:

\begin{tabular}{|c|c|c|c|c|c|c|c|}
\hline case $1 *$ : & & $\log l$ & $\log l^{\prime}$ & & . & $\log l^{\prime \prime}:$ & classical \\
\hline case 1: & $\log l$ & $\cdot \log l^{\prime}$ & . & . & . & $\log l^{\prime \prime}:$ & $\begin{array}{l}\text { symmetry broken, } \\
\text { coupled flow }\end{array}$ \\
\hline case 2: & $\log l$ & . & $\log l^{\prime}$ & & . & $\log l^{\prime \prime}:$ & $\begin{array}{l}\text { symmetry broken, } \\
\text { memory effects }\end{array}$ \\
\hline ase 3 & $\log l$ & $\cdot \log l$ & . & $\log l^{\prime}$ & $\log l^{\prime}$ & $\begin{array}{l}\log l^{\prime \prime}: \\
\log { }^{\prime \prime}\end{array}$ & classical \\
\hline
\end{tabular}

As was already pointed out in the Introduction, the separation between the pore and the fracture scales can be roughly approximated by

$$
\frac{l}{l^{\prime}}=O\left(\sqrt{\frac{\mathbf{K}_{p}}{\mathbf{K}_{f}}}\right) .
$$

Therefore, it should be remarked that for a given double porosity medium for which the permeabilities $\mathbf{K}_{p}$ and $\mathbf{K}_{f}$ are well defined, the macroscopic behaviour depends on the macroscopic length $l^{\prime \prime}$, only. The latter characterizes the excitation or the size of the appropriate macroscopic sample and it is generally difficult to be determined. Nevertheless, it can be roughly evaluated from the fracture Darcy law which is valid whatever the macroscopic description. This law is written (from A.22):

$$
\left\langle\mathbf{v}_{f}^{0}\right\rangle-\mathbf{v}_{s}^{0}=-\mathbf{K}_{f} \nabla^{\prime \prime} p_{f}^{0} .
$$

If $\mathbf{v}$ measures the Darcy relative velocity and $p$ stands for the macroscopic pressure drop, we have

$$
\mathbf{v}=O\left(\mathbf{k}_{f} \frac{p}{l^{\prime \prime}}\right)
$$


and $l^{\prime \prime}$ can be approximated by

$$
l^{\prime \prime}=\frac{\mathbf{K}_{f} p}{\mathbf{v}} .
$$

Therefore, the classification of the different macroscopic descriptions can be given in the form

$$
\begin{aligned}
& \frac{\mathbf{v} \sqrt{\mu}}{p}=O\left(\frac{\mathbf{K}_{p}}{\sqrt{\mathbf{K}_{f}}}\right): \text { case } 1 \text {, broken symmetry, coupled flow, } \\
& \frac{\mathbf{v} \sqrt{\mu}}{p}=O\left(\sqrt{\mathbf{K}_{p}}\right): \text { case } 2 \text {, broken symmetry, memory effects, } \\
& \frac{\mathbf{v} \sqrt{\mu}}{p}=O\left(\sqrt[4]{\mathbf{K}_{p} \mathbf{K}_{f}}\right): \text { case 3, classical description. }
\end{aligned}
$$

This classification is valid under the following conditions

$$
\mathbf{K}_{p} \ll \mathbf{K}_{f} \text { and } \frac{l^{\prime}}{l^{\prime \prime}}=O\left(\frac{\mathbf{v}}{p} \sqrt{\frac{\mu}{\mathbf{K}_{f}}}\right) \ll 1 .
$$

Let us now consider again the example already introduced in paper I (from (7)). For this particular reservoir, we obtained

$$
\frac{l}{l^{\prime}}=O\left(\sqrt{\frac{\mathbf{K}_{p}}{\mathbf{K}_{f}}}\right) \approx 5 \times 10^{-3}
$$

corresponding to $l \approx 10^{-3} \mathrm{~m}$ and $l^{\prime} \approx 2 \times 10^{-1} \mathrm{~m}$. Therefore, with respect to the value of $l^{\prime \prime}$, we obtain the following macroscopic descriptions:

$l^{\prime \prime} \approx 10^{4} \mathrm{~m}:$ case 1 , broken symmetry, coupled flow,

$l^{\prime \prime} \approx 40 \mathrm{~m}$ : case 2 , broken symmetry, memory effects,

$l^{\prime \prime} \approx 3 \mathrm{~m}$ : $\quad$ case 3 , classical description.

In conclusion, we can expect the memory effects for this reservoir in the range $20 \mathrm{~m} \leqslant l^{\prime \prime} \leqslant 1000 \mathrm{~m}$.

It should be also noted that our investigations are limited to short times in relation to the fracture time $t$. For longer times, i.e. times of order $T_{p}$, it is clear that the fractures behave like perfect drains. $p_{f}^{0}$ is independent of $\mathbf{x}^{\prime \prime}$ and the flow is described by modelling the single porous medium occupying $\Omega_{s+p}^{\prime}$.

As we show, the pore plus matrix domain exhibits memory effects in case 2 . But the result is different from that recalled in Auriault (1990), where a single porous medium saturated by a strongly viscous fluid is shown to behave at a macroscopic level like a single-phase viscoelastic medium. For that situation, the contrast between the elastic properties and the viscous property was of order 1: $|\mathbf{a}| T_{p} \mid$ $\mu=O(1)$. In our case $2,|\mathbf{a}| T_{f} / \mu=O(1)$ resulting in $|\mathbf{a}| T_{p} / \mu=O\left(\varepsilon^{2}\right)$. Here, the memory effects are due to the seepage through the pores, as can be seen from the set which gives $\mathbf{u}_{s}^{1}$. The modelling corresponding to case 2 appears to be the 
most fruitful, since it contains all other cases by either cancelling the memory effects or restoring the symmetry. It will therefore be a good choice when very large spectra of the sizes of pores and fractures prevent individualizing one of the modellings.

In case 3 , the pore plus matrix domain behaves like a single phase elastic medium and the fluid is ignored. We encounter here a situation described in Auriault (1990).

Finally, the investigation conducted in case 2 is a demonstration of one of the mechanisms invoked to interpret what is often referred to as the 'viscoelastic property' of fluid saturated, cracked solids (O'Connell and Budiansky, 1977; Toksöz et al., 1979; Johnson et al., 1979).

\section{Appendix: Case 2: Homogenization}

Introducing the developments presented in Section 2.1 in the set (8)-(17), we first see that the corresponding developments for the stresses are written in the form:

$$
\begin{aligned}
& \sigma_{s}=\varepsilon^{-2} \sigma_{s}^{-2}+\varepsilon^{-1} \sigma_{s}^{-1}+\sigma_{s}^{0}+\ldots, \\
& \sigma_{p}=\sigma_{p}^{0}+\varepsilon \sigma_{p}^{1}+\varepsilon^{2} \sigma_{p}^{2}+\ldots \\
& \sigma_{f}=\sigma_{f}^{0}+\varepsilon^{2} \sigma_{f}^{2}+\ldots
\end{aligned}
$$

And noticing $\nabla, \nabla^{\prime}$, and $\nabla^{\prime \prime}$, the gradient operators with respect to $\mathbf{x}, \mathbf{x}^{\prime}$ and $\mathbf{x}^{\prime \prime}$, respectively, and adopting similar notations for other operators, we obtain successively by equating like powers of $\varepsilon$ :

In the solid part

$$
\begin{aligned}
& \sigma_{s}^{-2}=\mathbf{a e}\left(\mathbf{u}_{s}^{0}\right), \\
& \sigma_{s}^{-1}=\mathbf{a}\left(\mathbf{e}\left(\mathbf{u}_{s}^{1}\right)+\mathbf{e}^{\prime}\left(\mathbf{u}_{s}^{0}\right)\right) \\
& \sigma_{s}^{0}=\mathbf{a}\left(\mathbf{e}\left(\mathbf{u}_{s}^{2}\right)+\mathbf{e}^{\prime}\left(\mathbf{u}_{s}^{1}\right)+\mathbf{e}^{\prime \prime}\left(\mathbf{u}_{s}^{0}\right)\right), \\
& \sigma_{s}^{1}=\mathbf{a}\left(\mathbf{e}\left(\mathbf{u}_{s}^{3}\right)+\mathbf{e}^{\prime}\left(\mathbf{u}_{s}^{2}\right)+\mathbf{e}^{\prime \prime}\left(\mathbf{u}_{s}^{1}\right)\right), \\
& \nabla \sigma_{s}^{-2}=0 \\
& \nabla \sigma_{s}^{-1}+\nabla^{\prime} \sigma_{s}^{-2}=0 \\
& \nabla \sigma_{s}^{0}+\nabla^{\prime} \sigma_{s}^{-1}+\nabla^{\prime \prime} \sigma_{s}^{-2}=0 \\
& \nabla \sigma_{s}^{1}+\nabla^{\prime} \sigma_{s}^{0}+\nabla^{\prime \prime} \sigma_{s}^{-1}=0 \\
& \nabla \sigma_{s}^{2}+\nabla^{\prime} \sigma_{s}^{1}+\nabla^{\prime \prime} \sigma_{s}^{0}=0
\end{aligned}
$$


In the pores

$$
\begin{aligned}
& \mathbf{v}_{p}^{0}=\frac{\partial \mathbf{u}_{p}^{0}}{\partial t}, \quad \mathbf{v}_{p}^{1}=\frac{\partial \mathbf{u}_{p}^{1}}{\partial t}, \quad \mathbf{v}_{p}^{2}=\frac{\partial \mathbf{u}_{p}^{2}}{\partial t}+\frac{\partial \mathbf{u}_{p}^{0}}{\partial T}, \\
& \sigma_{p}^{0}=-p_{p}^{0} \mathbf{I}+2 \mu \mathbf{e}\left(\mathbf{v}_{p}^{0}\right), \\
& \sigma_{p}^{1}=-p_{p}^{1} \mathbf{I}+2 \mu\left(\mathbf{e}\left(\mathbf{v}_{p}^{1}\right)+\mathbf{e}^{\prime}\left(\mathbf{v}_{p}^{0}\right)\right) \\
& \sigma_{p}^{2}=-p_{p}^{2} \mathbf{I}+2 \mu\left(\mathbf{e}\left(\mathbf{v}_{p}^{2}\right)+\mathbf{e}^{\prime}\left(\mathbf{v}_{p}^{1}\right)+\mathbf{e}^{\prime \prime}\left(\mathbf{v}_{p}^{0}\right)\right) \\
& \nabla \sigma_{p}^{0}=0 \\
& \nabla \sigma_{p}^{1}+\nabla^{\prime} \sigma_{p}^{0}=0 \\
& \nabla \sigma_{p}^{2}+\nabla^{\prime} \sigma_{p}^{1}+\nabla^{\prime \prime} \sigma_{p}^{0}=0 \\
& \nabla \mathbf{v}_{p}^{0}=0 \\
& \nabla \mathbf{v}_{p}^{1}+\nabla^{\prime} \mathbf{v}_{p}^{0}=0 \\
& \nabla \mathbf{v}_{p}^{2}+\nabla^{\prime} \mathbf{v}_{p}^{1}+\nabla^{\prime \prime} \mathbf{v}_{p}^{0}=0
\end{aligned}
$$

In the fractures:

$$
\begin{aligned}
& \mathbf{v}_{f}^{0}=\frac{\partial \mathbf{u}_{f}^{0}}{\partial t}, \quad \mathbf{v}_{f}^{1}=\frac{\partial \mathbf{u}_{f}^{1}}{\partial t}, \quad \mathbf{v}_{f}^{2}=\frac{\partial \mathbf{u}_{f}^{2}}{\partial t}+\frac{\partial \mathbf{u}_{f}^{0}}{\partial T}, \\
& \sigma_{f}^{0}=-p_{f}^{0} \mathbf{l}, \quad \sigma_{f}^{1}=-p_{f}^{1} \mathbf{l}+2 \mu \mathbf{e}^{\prime}\left(\mathbf{v}_{f}^{0}\right), \\
& \nabla^{\prime} \sigma_{f}^{0}=0, \quad \nabla^{\prime} \sigma_{f}^{1}+\nabla^{\prime \prime} \sigma_{f}^{0}=0, \\
& \nabla^{\prime} \mathbf{v}_{f}^{0}=0, \quad \nabla^{\prime} \mathbf{v}_{f}^{1}+\nabla^{\prime \prime} \mathbf{v}_{f}^{0}=0,
\end{aligned}
$$

On $\Gamma$ :

$$
\begin{aligned}
& \sigma_{s}^{-2} \mathbf{N}=0, \quad \sigma_{s}^{-1} \mathbf{N}=0, \quad \sigma_{s}^{0} \mathbf{N}=\sigma_{p}^{0} \mathbf{N}, \\
& \sigma_{s}^{1} \mathbf{N}=\sigma_{p}^{1} \mathbf{N}, \quad \sigma_{s}^{2} \mathbf{N}=\sigma_{p}^{2} \mathbf{N}, \\
& \mathbf{v}_{s}^{0}=\mathbf{v}_{p}^{0}, \\
& \mathbf{v}_{s}^{1}=\mathbf{v}_{p}^{1}, \\
& \mathbf{v}_{s}^{2}=\mathbf{v}_{p}^{2}, \\
& \mathbf{v}_{s}^{3}=\mathbf{v}_{p}^{3},
\end{aligned}
$$

On $\Gamma^{\prime}$ :

$$
\begin{aligned}
& p_{p}^{0}=p_{f}^{0}, \quad p_{p}^{1}=p_{f}^{1}, \\
& \left\langle\sigma_{s}^{-2}\right\rangle_{\Omega} \mathbf{N}^{\prime}=0, \quad\left\langle\sigma_{s}^{-1}\right\rangle_{\Omega} \mathbf{N}^{\prime}=0, \\
& \left(\left\langle\sigma_{s}^{0}\right\rangle_{\Omega}+\left\langle\sigma_{p}^{0}\right\rangle_{\Omega}\right) \mathbf{N}^{\prime}=\sigma_{f}^{0} \mathbf{N}^{\prime}, \\
& \left(\left\langle\sigma_{s}^{1}\right\rangle_{\Omega}+\left\langle\sigma_{p}^{1}\right\rangle_{\Omega}\right) \mathbf{N}^{\prime}=\sigma_{f}^{1} \mathbf{N}^{\prime}, \\
& \mathbf{v}_{f}^{0}-\left\langle\mathbf{v}_{s}^{0}\right\rangle_{\Omega}=\left\langle\mathbf{v}_{p}^{0}\right\rangle_{\Omega}, \quad \mathbf{v}_{f}^{1}-\left\langle\mathbf{v}_{s}^{1}\right\rangle_{\Omega}=\left\langle\mathbf{v}_{p}^{1}\right\rangle_{\Omega},
\end{aligned}
$$


A.1 THE SOLID DOMAIN AT THE PORE LEVEL (TWO FIRST APPROXIMATIONS)

Here we obtain similar results to those in paper I.

- Equation (A.3a) with (A.2a) and (A.12a) is an homogeneous problem for $\mathbf{u}_{s}^{0}$

$\Omega$ periodic, with respect to the variable $\mathbf{x}$. It gives $\mathbf{u}_{s}^{0}$ as $\mathbf{x}$ independent:

$$
\mathbf{u}_{s}^{0}=\mathbf{u}_{s}^{0}\left(\mathbf{x}^{\prime}, \mathbf{x}^{\prime \prime}, t, T\right) \text { and } \sigma_{s}^{-2}=0 .
$$

- Equation (A.3b) therefore simplifies in:

$$
\nabla \sigma_{s}^{-1}=0 .
$$

With (A.2b) and (A.12b), we obtain a linear problem for $\mathbf{u}_{s}^{1} \Omega$ periodic, as a function of $\mathbf{e}^{\prime}\left(\mathbf{u}_{s}^{0}\right)$ :

$$
\mathbf{u}_{s}^{1}=\xi(\mathbf{x}) \mathbf{e}^{\prime}\left(\mathbf{u}_{s}^{0}\right)+\hat{\mathbf{u}}_{s}^{1}\left(\mathbf{x}^{\prime}, \mathbf{x}^{\prime \prime}, t, T\right),
$$

where $\xi$ is a third-order tensor well defined by the boundary-value problem and $\hat{\mathbf{u}}_{s}^{1}$ an arbitrary $\mathbf{x}$ independent vector.

- Consider Equation (A.3c). Taking the volume average over $\Omega$ and using the periodicity of $\sigma_{s}^{0}$ together with (A.12c) and (A.6a), leads to

$$
\nabla^{\prime}\left\langle\sigma_{s}^{-1}\right\rangle_{\Omega}=0,
$$

where the averaged stress is given by

$$
\left\langle\sigma_{s}^{-1}\right\rangle_{\Omega}=\mathbf{c}^{\prime} \mathbf{e}^{\prime}\left(\mathbf{u}_{s}^{0}\right), \quad \mathbf{c}^{\prime}=\langle\mathbf{a}(\mathbf{I}+\mathbf{e}(\xi))\rangle_{\Omega} .
$$

As in paper I, $\mathbf{c}^{\prime}$ is an elastic tensor. Equation (A.18) with (A.15b) is therefore a well-posed elastic problem for $\mathbf{u}_{s}^{0} \Omega^{\prime}$ periodic. Since it is homogeneous, it is straightforward to obtain:

$$
\mathbf{u}_{s}^{0}=\mathbf{u}_{s}^{0}\left(\mathbf{x}^{\prime \prime}, t, T\right)
$$

Therefore, $\mathbf{u}_{s}^{1}$ appears as $\mathbf{x}$ independent:

$$
\mathbf{u}_{s}^{1}=\mathbf{u}_{s}^{1}\left(\mathbf{x}^{\prime}, \mathbf{x}^{\prime \prime}, t, T\right) \text { and } \sigma_{s}^{-1}=0,
$$

and (A.3c) reduces to

$$
\nabla \sigma_{s}^{0}=0 .
$$

\section{A.2 THE PORE DOMAIN}

The set (A.6a, A.5a, A.7a, and A.13a) represents a boundary-value problem for $\mathbf{v}_{p}^{0}$ and $p_{p}^{0}$ :

$$
\nabla \sigma_{p}^{0}=0, \quad \sigma_{p}^{0}=-p_{p}^{0} \mathbf{I}+2 \mu \mathbf{e}\left(\mathbf{v}_{p}^{0}\right), \quad \nabla \mathbf{v}_{p}^{0}=0, \quad \mathbf{v}_{p}^{0}=\mathbf{v}_{s}^{0} \text { on } \Gamma .
$$

The solution is obvious:

$$
\mathbf{v}_{p}^{0}=\mathbf{v}_{s}^{0}\left(\mathbf{x}^{\prime \prime}, t, T\right), \quad p_{p}^{0}=p_{p}^{0}\left(\mathbf{x}^{\prime}, \mathbf{x}^{\prime \prime}, t, T\right)
$$


A.3 THE SOLID DOMAIN AT THE PORE LEVEL (THIRD APPROXIMATION)

The set (A.3c, A.2c, A.12c) is a boundary-value problem for $\mathbf{u}_{s}^{2}$ :

$$
\nabla \sigma_{s}^{0}=0, \quad \sigma_{s}^{0}=\mathbf{a}\left(\mathbf{e}\left(\mathbf{u}_{s}^{2}\right)+\mathbf{e}^{\prime}\left(\mathbf{u}_{s}^{1}\right)+\mathbf{e}^{\prime \prime}\left(\mathbf{u}_{s}^{0}\right)\right), \quad \sigma_{s}^{0} \mathbf{N}=-p_{p}^{0} \mathbf{N} .
$$

$\mathbf{u}_{s}^{2}$ appears as a linear function of $\mathbf{e}^{\prime}\left(\mathbf{u}_{s}^{1}\right)+\mathbf{e}^{\prime \prime}\left(\mathbf{u}_{s}^{0}\right)$ and $p_{p}^{0}$ :

$$
\mathbf{u}_{s}^{2}=\xi(\mathbf{x})\left(\mathbf{e}^{\prime}\left(\mathbf{u}_{s}^{1}\right)+\mathbf{e}^{\prime \prime}\left(\mathbf{u}_{s}^{0}\right)\right)-\eta(\mathbf{x}) p_{p}^{0}+\hat{\mathbf{u}}_{s}^{2}\left(\mathbf{x}^{\prime}, \mathbf{x}^{\prime \prime}, t, T\right),
$$

where $\hat{\mathbf{u}}_{s}^{2}$ is an arbitrary additive $\mathbf{x}$ independent vector.

\section{A.4 THE PORE AND FRACTURE DOMAINS}

The set (A.6b, A.7b, A.5b, A.13b) is a classical Darcy problem which gives the relative velocity $\mathbf{v}_{p}^{1}-\mathbf{v}_{s}^{1}$ as a linear function of $\nabla^{\prime} p_{p}^{0}$ :

$$
\begin{aligned}
& \mathbf{v}_{p}^{1}-\mathbf{v}_{s}^{1}=-\mathbf{k}_{p}(\mathbf{x}) \nabla^{\prime} p_{p}^{0}, \\
& p_{p}^{1}=\delta \nabla^{\prime} p_{p}^{0}+\hat{p}_{p}^{1}\left(\mathbf{x}^{\prime}, \mathbf{x}^{\prime \prime}, t, T\right),
\end{aligned}
$$

where the tensor $\mathbf{k}_{p}$ and the vector $\delta$ are well defined by the boundary-value problem, and $\hat{p}_{p}^{1}$ is an added $\mathbf{x}$ independent arbitrary function.

- Equation (A.10a) immediately gives

$$
p_{f}^{0}=p_{f}^{0}\left(\mathbf{x}^{\prime \prime}, t, T\right) \text {. }
$$

- Equation (A.7c) is written

$$
\nabla \mathbf{v}_{p}^{2}+\nabla^{\prime} \mathbf{v}_{p}^{1}+\nabla^{\prime \prime} \mathbf{v}_{p}^{0}=0
$$

Let us integrate over $\Omega$ and use the periodicity of $\mathbf{v}_{p}^{2}$ with $\mathbf{v}_{p}^{2}=0$ on $\Omega_{s}$. This gives

$$
\nabla^{\prime}\left\langle\mathbf{v}_{p}^{1}\right\rangle_{\Omega}+n \nabla^{\prime \prime} \mathbf{v}_{p}^{0}+\frac{1}{|\Omega|} \int_{\Gamma} \mathbf{v}_{p}^{2} \mathbf{N}_{p} \mathrm{~d} S=0 .
$$

With the help of (A.13c), (A.19), and (A.20), we obtain, after regrouping the terms

$$
\nabla^{\prime}\left(\left\langle\mathbf{k}_{p}\right\rangle_{\Omega} \nabla^{\prime} p_{p}^{0}\right)=-\alpha^{\prime} \frac{\partial\left(\mathbf{e}^{\prime}\left(\mathbf{u}_{s}^{1}\right)+\mathbf{e}^{\prime \prime}\left(\mathbf{u}_{s}^{0}\right)\right)}{\partial t}-\beta^{\prime} \frac{\partial p_{p}^{0}}{\partial t} .
$$

If we consider (slow) harmonic movement with pulsation $\omega$, it becomes

$$
\nabla^{\prime}\left(\left\langle\mathbf{k}_{p}\right\rangle_{\mathbf{\Omega}} \nabla^{\prime} p_{p}^{0}\right)=-\alpha^{\prime} i \omega\left(\mathbf{e}^{\prime}\left(\mathbf{u}_{s}^{1}\right)+\mathbf{e}^{\prime \prime}\left(\mathbf{u}_{s}^{0}\right)\right)-\beta^{\prime} i \omega p_{p}^{0} .
$$

- The set (A.10b, A.9b, and A.16a) is again a classical Darcy problem which gives the relative velocity $\mathbf{v}_{f}^{0}-\mathbf{v}_{s}^{0}$ as a linear function of $\nabla^{\prime \prime} p_{f}^{0}$ :

$$
\nabla^{\prime} \sigma_{f}^{1}+\nabla^{\prime \prime} \sigma_{f}^{0}=0, \quad \sigma_{f}^{1}=-p_{f}^{1} \mathbf{I}+2 \mu \mathbf{e}^{\prime}\left(\mathbf{v}_{f}^{0}\right), \quad \mathbf{v}_{f}^{0}=\mathbf{v}_{s}^{0} \text { on } \Gamma^{\prime} .
$$

Therefore, we have

$$
\mathbf{v}_{f}^{0}-\mathbf{v}_{s}^{0}=-\mathbf{k}_{f}\left(\mathbf{x}^{\prime}\right) \nabla^{\prime \prime} p_{f}^{0}
$$




\section{A.5 THE PORE AND SOLID DOMAINS}

Equation (A.3d) reduces to

$$
\nabla \sigma_{s}^{1}+\nabla^{\prime} \sigma_{s}^{0}=0 .
$$

With the help of (A.12d), we obtain

$$
\sigma_{s}^{1} \mathbf{N}=\sigma_{p}^{1} \mathbf{N} \text { on } \Gamma,
$$

and by integration on $\Omega_{s}$ and use of (A.6b), we arrive at

$$
\nabla^{\prime}\left(\left\langle\sigma_{s}^{0}\right\rangle_{\Omega}-n p_{p}^{0} \mathbf{1}\right)=0
$$

From (A.19) it is easy to obtain the average of $\sigma_{s}^{0}$ on $\Omega$ :

$$
\left\langle\sigma_{s}^{0}\right\rangle_{\Omega}=\mathbf{c}^{\prime}\left(\mathbf{e}^{\prime}\left(\mathbf{u}_{s}^{1}\right)+\mathbf{e}^{\prime \prime}\left(\mathbf{u}_{s}^{0}\right)\right)-\alpha^{\prime} p_{p}^{0} .
$$

On the other hand, we have on $\Gamma^{\prime}$

$$
\left(\left\langle\sigma_{s}^{0}\right\rangle_{\Omega}-n p_{p}^{0} I\right) \mathbf{N}^{\prime}=-p_{f}^{0} \mathbf{N}^{\prime}
$$

and

$$
p_{p}^{0}=p_{f}^{0}\left(\mathbf{x}^{*}, t, T\right) \text {. }
$$

- The set (A.21b, A.23, A.24, A.15c, and A.14a) is a boundary-value problem for $\mathbf{u}_{s}^{1}$ and $p_{p}^{0}$ under harmonic excitation. These appear as linear functions of $\mathbf{e}^{\prime \prime}\left(\mathbf{u}_{s}^{0}\right)$ and $p_{f}^{0}$ :

$$
\begin{aligned}
& \mathbf{u}_{s}^{1}=\xi^{\prime}\left(\mathbf{x}^{\prime}\right) \mathbf{e}^{\prime \prime}\left(\mathbf{u}_{s}^{0}\right)-\eta^{\prime}\left(\mathbf{x}^{\prime}\right) p_{f}^{0}+\hat{\mathbf{u}}_{s}^{1}\left(\mathbf{x}^{\prime \prime}, t, T\right), \\
& p_{p}^{0}=v^{\prime}\left(\mathbf{x}^{\prime}\right) \mathbf{e}^{\prime \prime}\left(\mathbf{u}_{s}^{0}\right)+\tau^{\prime}\left(\mathbf{x}^{\prime}\right) p_{f}^{0} .
\end{aligned}
$$

But it is important to notice that the tensors $\xi^{\prime}, \eta^{\prime}, v^{\prime}$ and the scalar $\tau^{\prime}$ are here complex valued and $\omega$ dependent.

\section{A.6 THE MACROSCOPIC DESCRIPTION}

The macroscopic description is composed of two relations.

- The first is obtained from the momentum balances. Let us consider Equations (A.3e), (A.6c), and (A.12e):

$$
\nabla \sigma_{s}^{2}+\nabla^{\prime} \sigma_{s}^{1}+\nabla^{\prime \prime} \sigma_{s}^{0}=0, \quad \nabla \sigma_{p}^{2}+\nabla^{\prime} \sigma_{p}^{1}-\nabla^{\prime \prime} p_{p}^{0}=0, \quad \sigma_{s}^{2} \mathbf{N}=\sigma_{p}^{2} \mathbf{N} .
$$

Integrating the first on $\Omega_{s}$ and the second on $\Omega_{p}$, with the third gives

$$
\nabla^{\prime}\left\langle\sigma_{s}^{1}\right\rangle_{\Omega}+\nabla^{\prime \prime}\left\langle\sigma_{s}^{0}\right\rangle_{\Omega}+\nabla^{\prime}\left\langle\sigma_{p}^{1}\right\rangle_{\Omega}-n \nabla^{\prime \prime} p_{p}^{0}=0 .
$$

Now consider Equations (A.10b) and (A.12d):

$$
\nabla^{\prime} \sigma_{f}^{1}+\nabla^{\prime \prime} \sigma_{f}^{0}=0, \quad\left(\left\langle\sigma_{s}^{1}\right\rangle_{\Omega}+\left\langle\sigma_{p}^{1}\right\rangle_{\Omega}\right) \mathbf{N}^{\prime}=\sigma_{f}^{1} \mathbf{N}^{\prime} .
$$


Integrating the first on $\Omega_{f}^{\prime}$ and (A.25) on $\Omega_{s+p}^{\prime}$, with the second gives

$$
\nabla^{\prime \prime}\left\langle\left\langle\sigma_{s}^{0}\right\rangle_{\Omega \Omega^{\prime}}-\nabla^{\prime \prime}\left\langle n p_{p}^{0}\right\rangle_{\Omega^{\prime}}-n^{\prime} \nabla^{\prime \prime} p_{f}^{0}=0\right.
$$

or

$$
\nabla^{\prime \prime}\left(\mathbf{c}^{\prime \prime} \mathbf{e}^{\prime \prime}\left(\mathbf{u}_{s}^{0}\right)-\alpha^{\prime \prime} p_{f}^{0}\right)=0 .
$$

The tensors $\mathbf{c}^{\prime \prime}$ and $\alpha^{\prime \prime}$ are here complex-valued and $\omega$ dependent.

- The second macroscopic relation comes from mass balances. We start with Equations (A.11b) and (A.16b):

$$
\nabla^{\prime} \mathbf{v}_{f}^{1}+\nabla^{\prime \prime} \mathbf{v}_{f}^{0}=0, \quad \mathbf{v}_{f}^{1}-\left\langle\mathbf{v}_{s}^{1}\right\rangle_{\Omega}=\left\langle\mathbf{v}_{p}^{1}\right\rangle_{\Omega} .
$$

Integrating the first on $\Omega_{f}^{\prime}$ and, using the second, we obtain:

$$
\nabla^{\prime \prime}\left\langle\mathbf{v}_{f}^{0}\right\rangle_{\Omega^{\prime}}=-\frac{1}{\left|\Omega^{\prime}\right|} \int_{\Gamma^{\prime}} \mathbf{v}_{f}^{1} \mathbf{N}_{f} \mathrm{~d} S=-\frac{1}{\left|\Omega^{\prime}\right|} \int_{\Gamma^{\prime}}\left(\left\langle\mathbf{v}_{s}^{1}\right\rangle_{\Omega}+\left\langle\mathbf{v}_{p}^{1}\right\rangle_{\Omega}\right) \mathbf{N}_{f} \mathrm{~d} S .
$$

Finally, now using the Darcy laws (A.20) and (A.22) for the pores and the fractures leads to

$$
\nabla^{\prime \prime}\left(\left\langle\mathbf{k}_{f}\right\rangle_{\Omega^{\prime}} \nabla^{\prime \prime} p_{f}^{0}\right)=-\gamma^{\prime \prime} i \omega \mathbf{e}^{\prime \prime}\left(\mathbf{u}_{s}^{0}\right)-\beta^{\prime \prime} i \omega p_{f}^{0} .
$$

\section{References}

Auriault, J. L. and Boutin, C., 1992, Deformable porous media with double porosity. Quasi-statics. I, Transport in Porous Media 7, 63-82.

Auriault, J. L., 1990, Behaviour of porous saturated deformable media, in F. Darve (ed.), Geomaterials: Constitutive Equations and Modelling, Elsevier, Amsterdam, pp. 311-328.

Auriault, J. L., 1991, Heterogeneous media. Is an equivalent macroscopic description possible? Int. J. Engn. Sci., 29, 785-795.

Johnston, D. H., Toksöz, M. N., and Timur, A., 1979, Attenation of seismic waves in dry and saturated rocks: II. Mechanisms, Geophysics 44, 69I-711.

O'Connell, R. J. and Budiansky, B., 1977, Viscoelastic properties of fluid saturated cracked solids, $J$. Geophys. Res. 82, 5719-5735.

Toksöz, M. N., Johnston, D. H., and Timur, A., 1979, Attenation of seismic waves in dry and saturated rocks: I. Laboratory measurements, Geophysics 44, 681-690.

Van Golf-Racht, T. D., 1982, Fundamentals of Fractured Reservoir Engineering, Elsevier, New York. 